\title{
Análisis de contenido utilizando webQDA en el estudio de la comunicación de riesgos durante el COVID-19: El caso de Nueva Zelanda
}

\author{
Henny Luz Heredia Martínez', e Elizabeth Artmann'1 \\ ${ }^{1}$ Escola Nacional de Saúde Pública Sérgio Arouca Fundação Oswaldo Cruz, Brasil | \\ hennyluzhm@hotmail.com; bethartmann@gmail.com | https://orcid.org/0000-0002-4609-048; \\ https://orcid.org/0000-0002-8690-5964
}

\begin{abstract}
Resumen: Introducción: La Salud Global afronta uno de los mayores retos de la historia para controlar la propagación del COVID-19, cuya principal característica es la velocidad de contagio. Paralelamente, la pandemia y la respuesta han ido acompañadas de una enorme infodemia: el exceso de información (precisa o no). La comunicación de riesgos es un componente fundamental de cualquier respuesta de emergencia. Objetivo: Analizar los componentes de la comunicación de riesgos que sustentaron la experiencia desarrollada en Nueva Zelanda para la respuesta al COVID-19. Metodología: Investigación cualitativa, que busca entender cuáles y cómo fueron utilizados los componentes de la comunicación de riesgos en su contexto histórico-social, y por medio de sus registros, se procuró develar su efecto en el control del COVID-19 en Nueva Zelanda. El corpus de análisis fue constituido por 7 videos de acceso libre y 19 publicaciones del sitio web oficial del gobierno de Nueva Zelanda con declaraciones realizadas por la Primera Ministra Jacinda Ardern, durante el período de marzo/diciembre 2020. Todo el corpus documental fue sujeto al análisis de contenido utilizando webQDA. Resultados: Todos los componentes de la comunicación de riesgos sugeridos por la OMS fueron considerados en la experiencia de Nueva Zelanda, con un fuerte enfoque de participación de la comunidad/diversos actores y promoción de la solidaridad como valor central de la sociedad neozelandesa, a través del uso de mensajes claros y consistentes. La estrategia de comunicación dio relevancia a las decisiones basadas en la mejor evidencia científica, con una marcada promoción de las intervenciones no farmacológicas. Conclusiones. La investigación cualitativa y los CAQDAS como el webQDA son herramientas fundamentales para el estudio de temas relevantes como la comunicación de riesgos, con énfasis en el discurso político y sus implicaciones en el desarrollo de estrategias para controlar el COVID-19 y la infodemia.
\end{abstract}

Palabras clave: Covid-19; Comunicación de Riesgos; Infodemia; Análisis de Contenido; Nueva Zelanda; webQDA.

Content Analysis Using Webqda in the Study of Risk Communication During COVID19: The Case of New Zealand

\begin{abstract}
Introduction: Global Health is facing one of the greatest challenges in history to control the spread of COVID-19, whose main characteristic is the speed of contagion. At the same time, the pandemic and the response have been accompanied by an enormous infodemia: the excess of information (accurate or not). Risk communication (CR) is a critical component of any emergency response. Objective: to analyze the components of the CR that supported the experience developed in New Zealand for the response to COVID-19. Methodology: Qualitative research, which seeks to understand which and how the components of risk communication were used in their historical-social context, and through their records, we sought to reveal its effect on COVID-19 control in New Zealand. The corpus of analysis was constituted by 7 open-access videos and 19 publications from the official website of the New Zealand government with statements made by Prime Minister Jacinda Ardern, during the period March/December 2020. The entire documentary corpus was subjected to content analysis using webQDA. Results: All components of risk communication suggested by WHO were considered in the New Zealand experience, with a strong focus on community/diverse actors' involvement and promotion of solidarity as a core value of New Zealand society, through the use of clear and consistent messaging. The communication strategy gave relevance to decisions based on the best scientific evidence, with a marked promotion of non-pharmacological interventions. Conclusions: Qualitative research and CAQDAS such as webQDA are fundamental tools for the study of relevant topics such as risk communication, with emphasis on political speech and its implications in the development of strategies to control COVID-19 and the infodemic.
\end{abstract}

Keywords: Covid-19; Risk Communication; Infodemia; Content Analysis; New Zealand; webQDA. 


\section{Introducción}

La Salud Global afronta uno de los mayores retos de la historia para controlar la propagación del coronavirus (SARS-Cov-2- COVID-19), cuya principal característica es la velocidad de contagio. La pandemia del COVID-19 declarada en marzo de 2020 por la Organización Mundial de la Salud (OMS, 2020a), llegó acompañada de una 'infodemia' masiva (World Health Organization, 2020c) definida como una sobreabundancia de información (precisa o no) que dificulta que las personas encuentren fuentes confiables para tomar decisiones informadas (World Health Organization, 2020c). Este fenómeno tiene un mayor impacto en la era de las redes sociales porque el volumen de informaciones se multiplica exponencialmente (Zarocostas, 2020), llegando en segundos a gran parte de la población mundial (World Health Organization, 2020b) y con limitados controles para la difusión de información/desinformación.

La OMS estableció la Red de Información sobre Epidemias (EPI-WIN) (Zarocostas, 2020), un área estratégica que busca la "simplificación del conocimiento" a través de mensajes útiles, prácticos y comprensibles, que consideren las necesidades de las poblaciones vulnerables y que contribuyan al cambio del comportamiento masivo, aspecto central en la respuesta al COVID-19 (World Health Organization, 2020b, 2020a).

Dentro de las medidas de mitigación del COVID-19 utilizadas en los países destacan:

- Intervenciones no farmacológicas (Anderson et al., 2020; Garcia \& Duarte, 2020; Han et al., 2020): correcto lavado de manos; uso de mascarilla; distanciamiento social (incluyendo aislamiento de los casos, cuarentena a los contactos y práctica voluntaria de no frecuentar lugares para evitar aglomeraciones); limpieza de los espacios y superficies de forma rutinaria; restricción del funcionamiento de escuelas, universidades, establecimientos comerciales que prestan servicios no esenciales, eventos y transporte público.

- Políticas sociales para enfrentar la pandemia con reducción de daños (Han et al., 2020): estas incluyen estrategias de solidaridad social diferenciadas para generar condiciones que permitan a las poblaciones más vulnerables poder cumplir con el distanciamiento social.

- Preparación de los servicios de salud y la regulación de los recursos críticos (Anderson et al., 2020; Han et al., 2020): con la finalidad de aumentar el acceso a pruebas, rastreo y control de los casos a través de la Atención Primaria de Salud; garantizar la atención de los pacientes COVID-19 en instalaciones adecuadas; preparar y proteger a los profesionales de la salud; garantizar la continuidad del cuidado de los pacientes con otras patologías; entre otros.

- Comunicación transparente (Anderson et al., 2020; Garcia \& Duarte, 2020; Han et al., 2020; World Health Organization, 2020a): aspecto fundamental para que la población/sociedad civil conozca los riesgos y las decisiones que se están tomando en tiempo real, según las mejores evidencias disponibles. Esto promueve la confianza de la población y su participación activa en la protección.

Este conjunto de medidas ha implicado altos costos políticos y económicos como sacrificios personales (Calheta et al., 2020). Su materialización e impacto varían de un país a otro y la estrategia de comunicación utilizada ha sido central para garantizar la adhesión informada de la población, así como la efectividad de las medidas (Anderson et al., 2020; Han et al., 2020).

Para la OMS (2020b), la comunicación para la salud es una de las herramientas esenciales durante las emergencias en salud pública. Específicamente, la comunicación de riesgos tiene como objetivo mitigar, aliviar y gestionar los riesgos potenciales (Betsch, 2020). Es un componente fundamental de cualquier respuesta de emergencia, porque las intervenciones más importantes y efectivas son las que permiten comunicar de manera proactiva lo que se sabe y se desconoce, así como lo que se está haciendo para obtener más información, con el fin último de salvar vidas y minimizar las consecuencias adversas (World Health Organization, 2020d). 
En la comunicación de riesgos las opiniones de científicos y expertos técnicos son cruciales, aunque se basa esencialmente en el conocimiento actual y en mensajes rutinarios, estructurados, controlados y sistemáticos (Betsch, 2020; Heymann \& Shindo, 2020).

Una comunicación de riesgos efectiva debe incluir los siguientes componentes (Organización Mundial de la Salud, 2005; World Health Organization, 2017, 2020d, 2020a): confianza y credibilidad; anuncios tempranos; transparencia; involucramiento de la comunidad y planificación con tiempo. A pesar de que la comunicación de riesgos abarca todos los componentes básicos de la comunicación para la salud, se diferencia en la necesidad de velocidad y la dependencia de la confianza (World Health Organization, 2020d). El intercambio de información y opiniones entre expertos, líderes comunitarios o funcionarios y las personas en riesgo se da en tiempo real (World Health Organization, 2017). En los momentos de crisis, se exige de los líderes respuestas rápidas, confiables y transparentes (World Health Organization, 2017, 2020d).

En la respuesta al COVID-19 es fundamental la implementación de estrategias efectivas de comunicación de riesgos y participación comunitaria (CRPC) que contribuyan a que las personas en mayor riesgo puedan comprender y adoptar comportamientos de protección (World Health Organization, 2020d, 2020a). Así como, mejorar la comprensión de las amenazas (reales o potenciales) a la salud por parte de la población; tomar decisiones informadas para mitigar los riesgos; y mejorar la confianza en la medida en que las autoridades y los expertos escuchen y den respuesta a las necesidades de las personas (Organización Mundial de la Salud, 2020b; Porat et al., 2020; World Health Organization, 2020d).

Nueva Zelanda es uno de los países elogiados por la OMS como "ejemplo global" en la pandemia (World Health Organization, 2020e), porque ha logrado mantener bajo control el COVID-19. La prensa internacional también ha destacado la labor de la Primera Ministra Jacinda Ardern en la respuesta al COVID-19 (El País, 2020; elDiario.es, 2020; The New York Times, 2020). Este artículo tiene por objetivo analizar los componentes de la comunicación de riesgos que sustentaron la experiencia desarrollada en Nueva Zelanda para la respuesta al COVID-19, durante el período de marzo a diciembre de 2020. Por tratarse de una investigación más amplia que está en desarrollo, se presentarán los resultados parciales.

\section{Metodología}

Se trata de una investigación cualitativa que buscó comprender la producción de fenómenos simbólicos, su papel social, efectos y significados sobre los componentes de la comunicación de riesgos que sustentaron la experiencia desarrollada en Nueva Zelanda. En este enfoque aparecen en el análisis los actores, los valores de los investigadores, la subjetividad, los hechos y sus significados, sin desconsiderar el conflicto (Minayo, 2004). De acuerdo a esta perspectiva, se realizó un estudio interpretativo para entender cuáles y cómo fueron utilizados los componentes de la comunicación de riesgos en su contexto histórico-social, y por medio de sus registros, se procuró develar su efecto en el control del COVID-19 en Nueva Zelanda.

El corpus de análisis fue constituido por 7 videos de acceso libre y 19 publicaciones del sitio web oficial del gobierno de Nueva Zelanda ${ }^{1}$ con declaraciones realizadas por la Primera Ministra Jacinda Ardern, durante el período de marzo a diciembre de 2020. Una vez realizada la trascripción de los videos, este contenido y el de las publicaciones fue traducido al español.

\footnotetext{
1 Sitio web oficial del gobierno de Nueva Zelanda; Web: https://www.beehive.govt.nz/. Cada publicación
} cita los trechos textuales de lo que fue dicho por la Primera Ministra Jacinda Ardern. 
Todo el corpus documental fue sujeto al análisis de contenido utilizando webQDA2 (Costa \& Amado, 2018), software de análisis de datos cualitativos asistido por computadora (siglas en inglés CAQDAS).

Bardin (1996) define el análisis de contenido como un conjunto de técnicas para la interpretación de las comunicaciones presentes en situaciones cotidianas. Este análisis considera la presencia o ausencia de características de contenido, en un fragmento del mensaje. Las inferencias del contenido de un texto son replicables a su contexto social, esto es posible por la realización de un trabajo práctico, objetivo y sistemático (Bardin, 1996), a través del cual los investigadores pueden explorar y comprender el tema estudiado. El análisis de contenido es parte integrante de la investigación en comunicación (Krippendorff, 1989) y a través de sus técnicas busca describir el contenido emitido (hablado/textos) en el proceso de comunicación (Bardin, 1996; Minayo, 2004).

Operacionalmente se utilizaron las etapas del análisis temático de contenido propuestas por Bardin (1996) y Minayo (2004): pre-análisis; exploración del material, con el análisis del texto a partir de la categorización; y tratamiento e interpretación de los resultados. Las dos investigadoras por separado realizaron una lectura fluctuante, repetida y profunda del corpus documental constituido por 26 archivos, organizados e identificados por orden cronológico y asunto tratado, gravados en archivos separados.

Se utilizó un enfoque de análisis marco, creando temas predefinidos para la estructura de codificación. El marco de codificación se guió por construcciones teóricas de la literatura y revisiones previas sobre los componentes de la comunicación de riesgos y sus beneficios en la respuesta al COVID-19 (Bórquez et al., 2020; Organización Mundial de la Salud, 2005; Porat et al., 2020; World Health Organization, 2020d, 2020a). Tras las primeras lecturas exploratorias, cada una de las investigadoras realizó por separado la codificación inicial con base en las categorías establecidas por la OMS (2005; World Health Organization, 2020c, 2020a) y en las directrices propuestas por Porat et al. (2020). Los códigos se integraron de forma gradual en las categorías mediante la comparación entre transcripciones. Los temas y categorías emergentes fueron incluidos mediante discusión consensuada por las dos investigadoras. Así, el proceso de categorización terminó siendo mixto (Coutinho, 2013).

Luego, se desarrolló el análisis temático que consiste en buscar los núcleos de significado en los textos, para explorar temas y categorías emergentes relacionadas con los objetivos del estudio que se infirieron de los datos. La categorización o clasificación se realizó seleccionando los extractos más relevantes de los textos, a partir de la diferenciación de los elementos constitutivos de ese conjunto, y después se reagruparon por analogía, se describieron los resultados y se buscaron inferencias e interpretaciones (Bardin, 1996; Minayo, 2004). Las diferencias encontradas en el proceso de categorización fueron discutidas y consensuadas por las investigadoras. Finalmente, se resumió la codificación y se establecieron conexiones entre temas relacionados. Para asegurar la confiabilidad, se propició una discusión continua y reflexiva sobre los resultados con el equipo de investigación.

Los temas y categorías de análisis identificadas en el corpus documental fueron incluidos en el software webQDA, utilizando la funcionalidad "códigos árbol", así como los indicadores producto del análisis de contenido. También se generó la nube de palabras.

\footnotetext{
${ }^{2}$ webQDA - Software de Análise Qualitativa de Dados; Web: https://www.webqda.net/
} 


\section{Resultados y Discusión}

Todos los componentes de la comunicación de riesgos sugeridos por la OMS (Organización Mundial de la Salud, 2005) fueron considerados en la experiencia de Nueva Zelanda (Tabla 1), con un fuerte enfoque de participación de la comunidad/diversos actores y la promoción de la solidaridad como valor central de la sociedad neozelandesa, en mensajes claros y consistentes (Porat et al., 2020) (Tabla 1). En la nube de palabras (Fig. 1), el centro es "todos".

El segundo tema (Tabla 1) obtuvo el mayor número de referencias y fuentes. Gobernanza y liderazgo fue la categoría más referenciada. El gobierno promovió varias acciones para atender a las poblaciones vulnerables (Tabla 1), aspecto este fundamental para que las personas con más carencias pudieran efectivamente cumplir con las mediadas de distanciamiento social (Han et al., 2020).

La estrategia de comunicación dio relevancia a las decisiones basadas en la mejor evidencia científica, con una marcada promoción de las intervenciones no farmacológicas (Anderson et al., 2020; Garcia \& Duarte, 2020; Han et al., 2020), así como al uso integrado de las redes sociales para mantener informada a la población, como para atender demandas específicas (p.e. salud mental) (Bórquez et al., 2020).

Las limitaciones, incertidumbres y errores se comunicaban de forma transparente con amplia participación de los medios de comunicación (Anderson et al., 2020; Garcia \& Duarte, 2020; Han et al., 2020; World Health Organization, 2020a). Uno de los momentos más críticos fue la renuncia del Ministro de Salud, debido a que faltó a las reglas de distanciamiento social, sin embargo, la decisión fue de que continuara con algunas condiciones, porque la prioridad era "salvar vidas" (Tabla 1). El sistema de salud y su sistema de información fueron priorizados para poder garantizar el acceso a las pruebas, como la atención de los casos sospechosos y los enfermos (Anderson et al., 2020; Han et al., 2020). De esta forma Nueva Zelanda logró disminuir en 2020 la circulación del virus sin vacunas.

Tabla 1. Temas y categorías de análisis según frecuencia de referencias y fuentes.

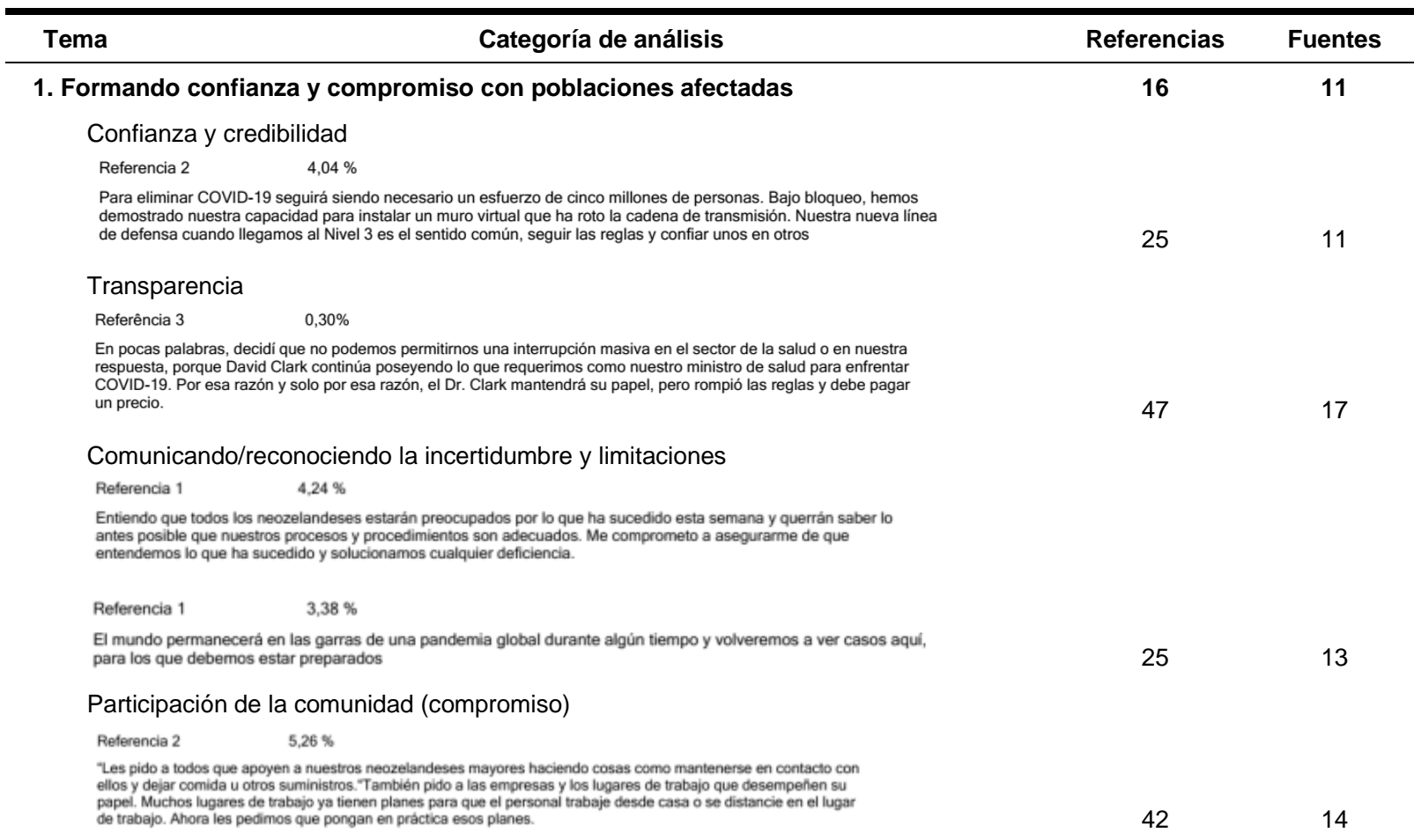




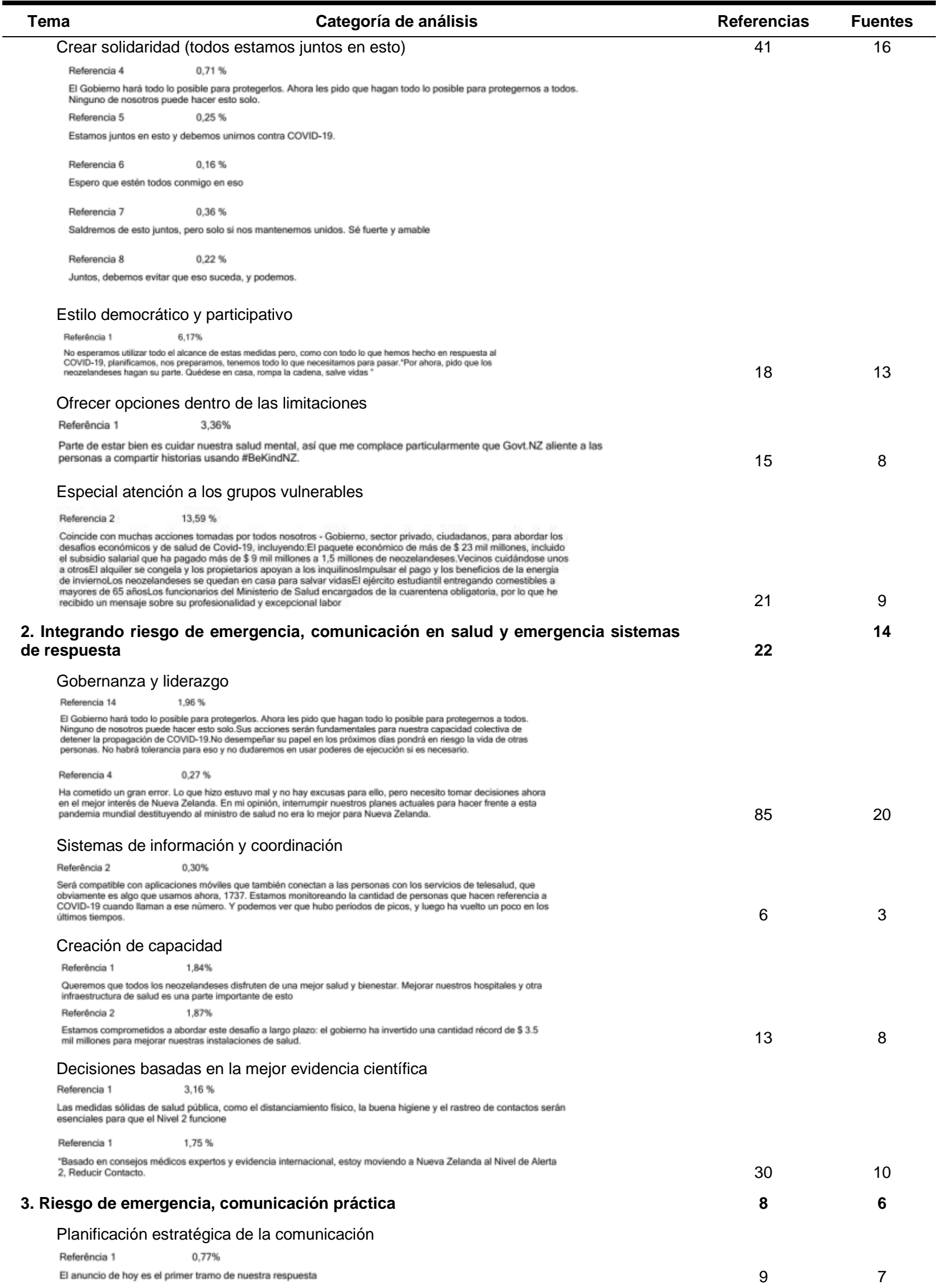




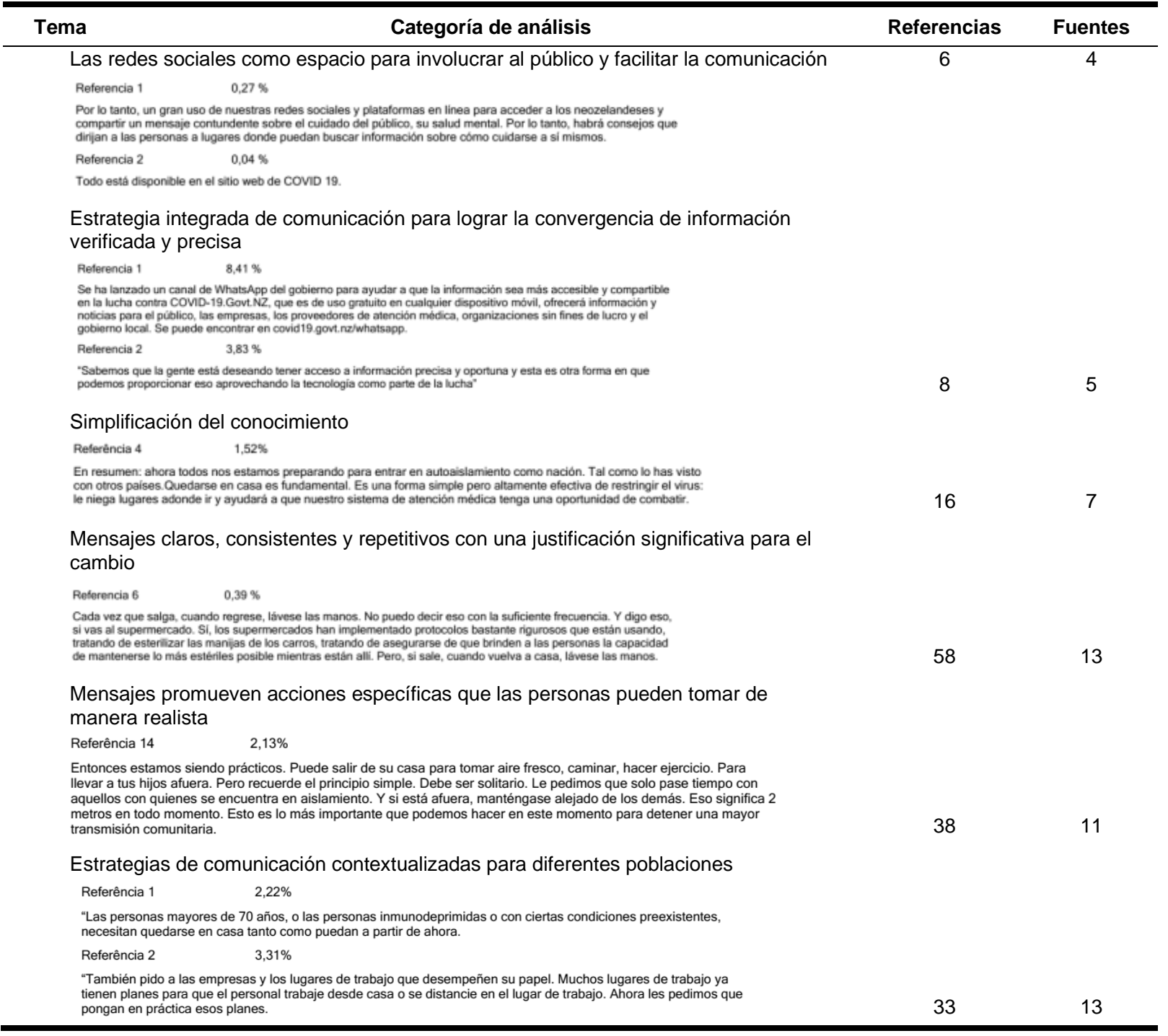

La nube de palabras (Fig. 1.) además de "todos hay destaque para las palabras; personas; Nueva Zelanda; salud; nivel; COVID-19. Así como algunos verbos conjugados en la primera persona del plural (estamos, hemos, podemos), utilizados en la estrategia de comunicación para denotar la participación y la unión de todos y todas en la respuesta al COVID-19.

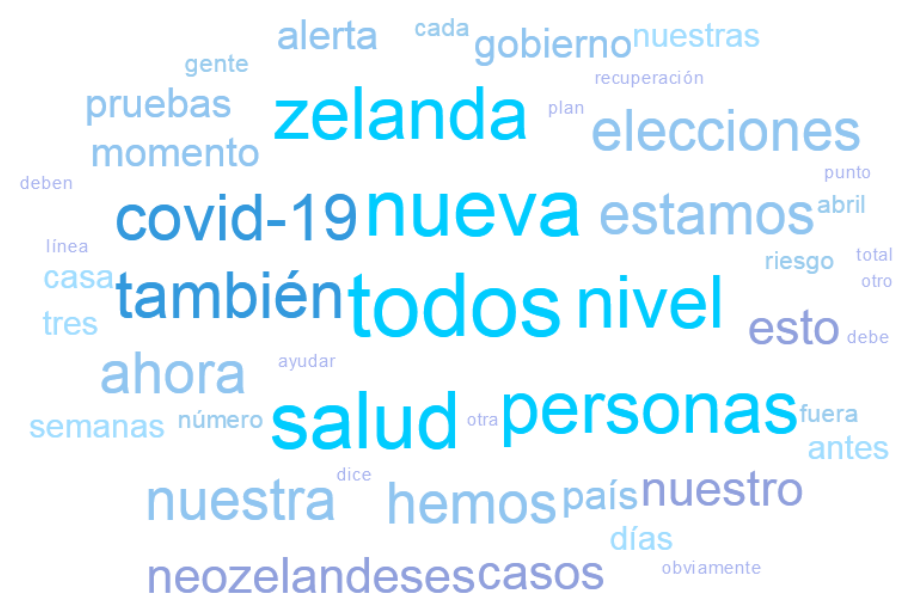

Fig. 1. Nube de palabras más frecuentes. 


\section{Conclusiones}

En tiempos de pandemia e infodemia (Organización Mundial de la Salud, 2020a; World Health Organization, 2020c) la salud colectiva además de responder a una situación sin precedentes, el COVD-19, tiene el desafío contemporáneo de lidiar con el campo de la información digital, desde el cual globalmente se generan y transmiten información/desinformación (World Health Organization, 2020b; Zarocostas, 2020). En este contexto, la investigación cualitativa y los CAQDAS como el webQDA son herramientas fundamentales para el estudio de temas relevantes como la comunicación de riesgos, con énfasis en el discurso político y sus implicaciones en el desarrollo de estrategias para controlar el COVID-19 y la infodemia. Estas metodologías tienen un gran potencial para contribuir en la sistematización de las diferentes acciones desarrolladas por los países durante la pandemia, aportando al conocimiento sobre cómo éstas fueron comunicadas, cuáles fueron las prioridades establecidas y su impacto en los resultados obtenidos. Además permiten optimizar la organización y procesamiento de grandes cantidades de información; incluir diferentes formatos de información que han surgido en la era de digital; promover al trabajo colaborativo de investigadores; entre otros (Costa \& Amado, 2018).

La experiencia de Nueva Zelanda durante el año 2020 demuestra que el país preparó una respuesta integral al COVID-19, con una fuerte política de comunicación de riesgos y participación comunitaria basada en los componentes sugeridos por la OMS (Organización Mundial de la Salud, 2005; World Health Organization, 2017, 2020d, 2020a); acompañada de políticas sociales a favor de los más vulnerables y planes para rescatar la economía nacional. Con lo que han podido responder a sus mayores prioridades: salvar el mayor número de vidas y resguardar la salud de la población.

\section{Agradecimientos}

Este trabajo fue realizado con apoyo del Programa Innova de la Fundación Oswaldo Cruz.

\section{Referencias}

Anderson, R. M., Heesterbeek, H., Klinkenberg, D., \& Hollingsworth, T. D. (2020). How will countrybased mitigation measures influence the course of the COVID-19 epidemic? The Lancet, 395(10228), 931-934. https://doi.org/10.1016/S0140-6736(20)30567-5

Bardin, L. (1996). Análise de conteúdo (2a). Edições 70.

Betsch, C. (2020). How behavioural science data helps mitigate the COVID-19 crisis. Nature Human Behaviour, 4(5), 438-438. https://doi.org/10.1038/s41562-020-0866-1

Bórquez, B., Luengo-Charath, M. X., Anguita M., V., Bascuñán R., M. L., Pacheco M., I. M., Michaud Ch., P., \& Vacarezza Y., R. (2020). Uso y difusión responsable de la información en pandemia: Un imperativo ético. Revista Chilena de Pediatría, 91(5), 794. https://doi.org/10.32641/rchped.v91i5.2420

Calheta, B., Fontes, B., Biagio, D., Rodrigues, I., Medeiros, K., Salatino, L., Fialho, M. C., \& Paixão, M. (2020). Boletim $N^{\circ} 06$ Direitos na pandemia mapeamento e análise das normas jurídicas de resposta à COVID-19 no Brasil. Lockdown à brasileira (Boletim Direitos na pandemia). CEPEDISA e CONECTAS Direitos Humanos.

Costa, A. P., \& Amado, J. (2018). Análise de Conteúdo suportada por software (1aed. Oliveira de Azeméis). Ludomedia.

Coutinho, C. M. P. (2013). Metodologia de investigaçao em ciencias sociais e humanas: Teoria e prática. Almedina. 
El País. (2020). El Gobierno de Nueva Zelanda confina a la mayor ciudad del país tras detectar cuatro contagios. El País. https://elpais.com/sociedad/2020-08-11/el-gobierno-de-nuevazelanda-confina-a-la-mayor-ciudad-del-pais-tras-detectar-cuatro-contagios.html

elDiario.es. (2020). "Sean fuertes, sean amables": Jacinda Ardern vuelve a sobresalir en su segunda crisis como primera ministra de Nueva Zelanda. elDiario.es. https://news.un.org/en/story/2020/08/1069842

Garcia, L. P., \& Duarte, E. (2020). Intervenções não farmacológicas para o enfrentamento à epidemia da COVID-19 no Brasil. Epidemiologia e Serviços de Saúde, 29(2). https://doi.org/10.5123/S1679-49742020000200009

Han, E., Tan, M. M. J., Turk, E., Sridhar, D., Leung, G. M., Shibuya, K., Asgari, N., Oh, J., GarcíaBasteiro, A. L., Hanefeld, J., Cook, A. R., Hsu, L. Y., Teo, Y. Y., Heymann, D., Clark, H., McKee, M., \& Legido-Quigley, H. (2020). Lessons learnt from easing COVID-19 restrictions: An analysis of countries and regions in Asia Pacific and Europe. The Lancet, 396(10261), 1525-1534. https://doi.org/10.1016/S0140-6736(20)32007-9

Heymann, D. L., \& Shindo, N. (2020). COVID-19: What is next for public health? The Lancet, 395(10224), 542-545. https://doi.org/10.1016/S0140-6736(20)30374-3

Krippendorff, K. (1989). Content analysis. In E. Barnouw, G. Gerbner, W. Schramm, T. L. Worth, \& L. Gross (Orgs.), International Encyclopedia of Communication (Vol. 1, p. 403-407). Oxford University Press.

Minayo, M. C. de S. (2004). O desafio do conhecimento: Pesquisa qualitativa em saúde. Hucitec.

Organización Mundial de la Salud. (2005). Normas de comunicación de brotes epidémicos de la OMS (OMS/CDS/2005.28; p. 10). OMS.

Organización Mundial de la Salud. (2020a). Alocución de apertura del Director General de la OMS en la rueda de prensa sobre la COVID-19 celebrada el 11 de marzo de 2020. OMS. https://www.who.int/es/director-general/speeches/detail/who-director-general-s-openingremarks-at-the-media-briefing-on-covid-19---11-march-2020

Organización Mundial de la Salud. (2020b). COVID-19 Orientaciones para comunicar sobre la enfermedad por el coronavirus 2019. Guía para líderes (p. 16). OMS.

Porat, T., Nyrup, R., Calvo, R. A., Paudyal, P., \& Ford, E. (2020). Public Health and Risk Communication During COVID-19-Enhancing Psychological Needs to Promote Sustainable Behavior Change. Frontiers in Public Health, 8, 573397. https://doi.org/10.3389/fpubh.2020.573397

The New York Times. (2020). Jacinda Ardern Sold a Drastic Lockdown With Straight Talk and Mom Jokes. The New York Times. https://www.nytimes.com/2020/05/23/world/asia/jacinda-arderncoronavirus-new-zealand.html

World Health Organization (Org.). (2017). Communicating risk in public health emergencies: $A$ WHO guideline for emergency risk communication (ERC) policy and practice. WHO.

World Health Organization (Org.). (2020a). Global Risk Communication and Community Engagement Strategy. WHO.

World Health Organization (Org.). (2020b). Infodemic Management-Infodemiology. Ad-Hoc Technical Consultation on Managing the COVID-19 Infodemic. WHO.

World Health Organization. (2020c). Novel Coronavirus(2019-nCoV) Situation Report-13. Data as reported by 2 February 2020 (Situation Report-13; p. 7). WHO.

World Health Organization. (2020d). Risk communication and community engagement readiness and response to coronavirus disease (COVID-19) (Interim guidance 19 March 2020; p. 5). WHO.

World Health Organization. (2020e). WHO chief points to 'green shoots of hope' in COVID-19 pandemic. WHO. https://news.un.org/en/story/2020/08/1069842

Zarocostas, J. (2020). How to fight an infodemic. The Lancet, 395(10225), 676. https://doi.org/10.1016/S0140-6736(20)30461-X 